\section{Pylephlebitis secondary to strangulated umbilical hernia with small bowel ischemia}

\author{
Ennio Bruschi, ${ }^{1}$ Giovanna Graziani, ${ }^{1}$ \\ Maurizio Vergendo, ${ }^{1}$ Antonello Conte, ${ }^{2}$ \\ Massimo Valentino' \\ 'Radiology Department, Sant'Antonio \\ Abate Hospital; 2Surgery Department, \\ Sant'Antonio Abate Hospital, Tolmezzo, \\ Italy
}

\section{Abstract}

Pylephlebitis is a septic thrombophlebitis of the portal venous system that infrequently complicates small bowel infarction. We present a case of pylephlebitis with portomesenteric vein gas bubbles secondary to small bowel ischemia caused by a strangulated umbilical hernia, diagnosed on computed tomography (CT) and confirmed in the operating theater. This case is an example of the usefulness of CT in early recognition of suggestive radiologic findings of pylephlebitis associated with intestinal ischemia for prompt treatment of the patient.

\section{Introduction}

We report a case of portomesenteric venous gas combined with band-like pneumatosis, a diagnosis based on computed tomography (CT) evidence of both findings. We performed an emergency laparotomy for suspected acute bowel ischemia, which was confirmed by the operative findings.

\section{Case Report}

A 58-year-old man came to Sant'Antonio Abate Hospital with severe abdominal pain, tachycardia, hypotension and hypoxia. His medical history included hypertension, congestive heart failure, atrial fibrillation, replacement of the ascending aorta, HCV-related liver disease and obesity. Laboratory studies revealed leukocytosis (white cell count of $12.13 \times 10^{3} / \mu \mathrm{L}$ vs normal range $4.20-11.0 \times 10^{3} / \mu \mathrm{L}$ ), with normal hemoglobin level and platelet count. Liver enzyme levels were normal. Total bilirubin level was normal and direct bilirubin level was elevated $(0.56 \mathrm{mg} / \mathrm{dL} v s$ normal range $0-0.40 \mathrm{mg} / \mathrm{dL})$. International normalized ratio was 10.52 (normal range 2-4.5) and C-reactive protein (CRP) was $69 \mathrm{mg} / \mathrm{L}$ (normal range $0-5 \mathrm{mg} / \mathrm{L}$ ).
Ultrasound examination, which was performed in the emergency department, revealed absence of fluid in the abdominal cavity, although the examination was limited because of the patient's body type and the presence of abundant meteorism.

Computed tomography examination, performed before and after intravenous administration of contrast media ( $120 \mathrm{~mL}$ of Ioexolo 350 $\mathrm{mgl} / \mathrm{mL}$, Omnipaque 350; Schering, BerlinWedding, Germany) showed evidence of several gas bubbles within superior mesenteric vein and its branches diffusely extended to the intrahepatic portal branches (Figures 1 and 2). Signs of pneumatosis parietalis were also demonstrated (Figures 2 and 3). In the umbilical region, a strangulated small bowel hernia was demonstrated, with dilatation of multiple proximal small bowel loops (Figure 4), which showed thickened and contrast-enhancing walls. Diagnosis of small bowel ischemia secondary to strangulated hernia with pylephlebitis was made on the basis of CT findings. The patient underwent surgical treatment with resection of a small bowel loop and latero-lateral ileal anastomosys was made. The patient gradually recovered with reduction of white blood cell (WBC) levels and CRP.

\section{Discussion}

Pylephlebitys is a septic thrombophlebitis of the portal venous system, usually secondary to an infection in the region drained by the portal system or in the structures contiguous to the portal vein. Pylephlebitis begins with thrombophlebitis of the small veins that drain the infected area. ${ }^{1}$ There are a few articles that describe the imaging presentation of pylephlebitys, an infrequent but known complication of small bowel infarction. ${ }^{2-7}$ Portomesenteric vein gas is most commonly caused by mesenteric ischemia but may have a variety of other causes. Main causes of this complication include: complicated diverticulitis, appendicitis, urinary infections, pelvic infections, biliary diseases, inflammatory bowel diseases, necrotizing pancreatitis, infections with unknown source (Table 1).

Primary factors that favor the development of this pathologic entity are intestinal wall alterations, bowel distention, and sepsis. Although the pathogenesis of portomesenteric venous gas and pneumatosis intestinalis in acute bowel ischemia has not been established, it may be related to the ulcers and ruptures in the continuity of the bowel wall in necrotic mucosa where normal resistance to intraluminal gas pressure is lost. ${ }^{7}$ Extension of the thrombophlebitis into larger veins leads to septic thrombophlebitis of the mesenteric vein, which can extend further to involve the
Correspondence: Ennio Bruschi, Radiology Department, Sant'Antonio Abate Hospital, via Morgagni Giobatta 18, 33028 Tolmezzo, Italy. Tel. +39.347 .9904714 - Fax: +39.0433 .48833 .

E-mail: bruschie73@gmail.com

Key words: pylephlebitis, strangulated umbilical hernia, small bowel ischemia.

Contributions: the authors contributed equally.

Conflict of interests: the authors declare no potential conflict of interests.

Received for publication: 21 March 2013.

Revision received: 29 May 2013.

Accepted for publication: 30 May 2013

This work is licensed under a Creative Commons Attribution 3.0 License (by-nc 3.0).

(C) Copyright E. Bruschi et al., 2013

Licensee PAGEPress, Italy

Emergency Care Journal 2013; 9:e12

doi:10.4081/ecj.2013.e12

portal vein. Symptoms and diagnostic imaging findings of small bowel ischemia have been described. Pylephlebitis is a severe clinical entity, having a mortality of $11-32 \%$. Clinical manifestations are usually confusing, varying from asymptomatic through minimal symptoms related to the primary infection site, to acute abdomen. In the subacute form of venous mesenteric thrombosis, patients may have abdominal pain for days or weeks, without bowel infarction. In $88 \%$ of patients there is bacteriemia, with $E$. coli and $B$. fragilis as the main pathogens. Liver involvement can cause hepatomegaly, elevation of liver enzyme levels, abscesses and jaundice. Seldom, there is a hypercoagulable state associated. ${ }^{8}$ Imaging findings of pylephlebitis are related to venous involvement with presence of intravascular thrombi or air, the primary source of infection and intrahepatic anomalies, such as unopacified branches of the portal vein, transient parenchymal attenuation differences and intrahepatic abscesses. ${ }^{8}$ At CT, portal vein gas appears as tubular areas of decreased attenuation in the liver, predominantly in the left lobe. These low-attenuation areas are caused by the accumulation of gas in the intrahepatic portal veins, where it is carried by centrifugal blood to the hepatic periphery. Portal vein gas can be visualized at conventional radiography, but substantial amounts must be present for detection. ${ }^{7}$ The radiographic criterion for portal vein gas is a branching area of low attenuation extending to $2 \mathrm{~cm}$ of the liver capsule. ${ }^{3}$ Intrahepatic portal vein gas must be differentiated from air in the biliary tree (pneumobilia). In latter condition, the air is located centrally 
(i.e. it does not extend to $2 \mathrm{~cm}$ of the liver capsule) and a confluence of air is seen in the common hepatic duct. Pneumobilia also has a left lobe predilection at CT due to its more ventral location. Gas in the small mesenteric veins appears as tubular or branched areas of decreased attenuation in the mesenteric border of the bowel. At CT, pneumatosis may manifest with small isolated gas bubbles within an ischemic bowel wall or as broad rims of air dissecting the entire bowel wall into two layers. ${ }^{3}$ The reported specificities of pneumatosis and portal venous gas for acute bowel ischemia usually approach $100 \%{ }^{3}$ In agreement with the literature, ${ }^{7}$ not only is the presence of full-thickness bowel wall necrosis, but also the extent of transmural bowel infarction that determines the clinical outcome of affected patients. Computed tomography findings of pneumatosis intestinalis and portomesenteric venous gas due to bowel ischemia do not generally allow prediction of transmural bowel infarction, because they may be observed in patients with only partial ischemic bowel wall damage. The clinical outcome of patients with bowel ischemia with these CT findings seems to depend mainly on the severity and extent of their underlying disease. Pylephlebitis remains an entity with high morbidity and mortality, seldom having subtle signs and symptoms. Still, early recognition of suggestive radiologic findings with the combination of findings of small bowel infarction and multiple venous thrombosis and/or gas bubbles in the region drained by the portal system, thanks to modern imaging modalities, may improve the prognosis of patients.

\section{Conclusions}

Computed tomography findings of pneumatosis intestinalis and portomesenteric venous gas due to bowel ischemia do not generally allow prediction of transmural bowel infarction, because they may be observed in patients with only partial ischemic bowel wall damage. The clinical outcome of patients with bowel ischemia with these CT findings seems to depend mainly on the severity and extent of their underlying disease. ${ }^{6}$ The presence of portomesenteric venous gas has been previously indicative of an unfavorable clinical outcome, with a poor prognosis in patients having bowel ischemia. We agree with the theory that it is not only the pneumatosis, the severity of bowel ischemia and the presence of a full-thickness bowel wall necrosis, but also the extent of transmural bowel infarction that mainly determine the clinical outcome of affected patients. ${ }^{6}$ However, urgent laparotomy is mandatory in patients at risk of ischemic bowel, with CT findings of portomesenteric venous gas and bandlike pneumatosis.
Table 1. Reported causes of portomesenteric venous gas.

Necrotic bowel

SMA syndrome

Cholangitis

Organ transplant (liver, kidney, bone marrow)

Inflammatory bowel disease

Bowel obstruction

Acute suppurative cholecystitis

Graft-us-host reaction

Peptic ulcer

Blunt abdominal trauma

Colonic diverticulitis

Pulmonary disease (chronic obstructive pulmonary disease, bronchopneumonia, asthma)

Acute necrotizing pancreatitis

Child abuse

Colitis steroid and cytostatic therapy

Intraperitoneal tumor

Barotraumas

Abdominal tuberculosis

Seizures

Severe enteritis

ERCP

Intra-abdominal abscess

Colonoscopy

Retroperitoneal abscess

Barium enema

Gangrene

Portal phlebitis

SMA, superior mesenteric artery syndrome; ERCP, endoscopic retrograde cholangio-pancreatography.
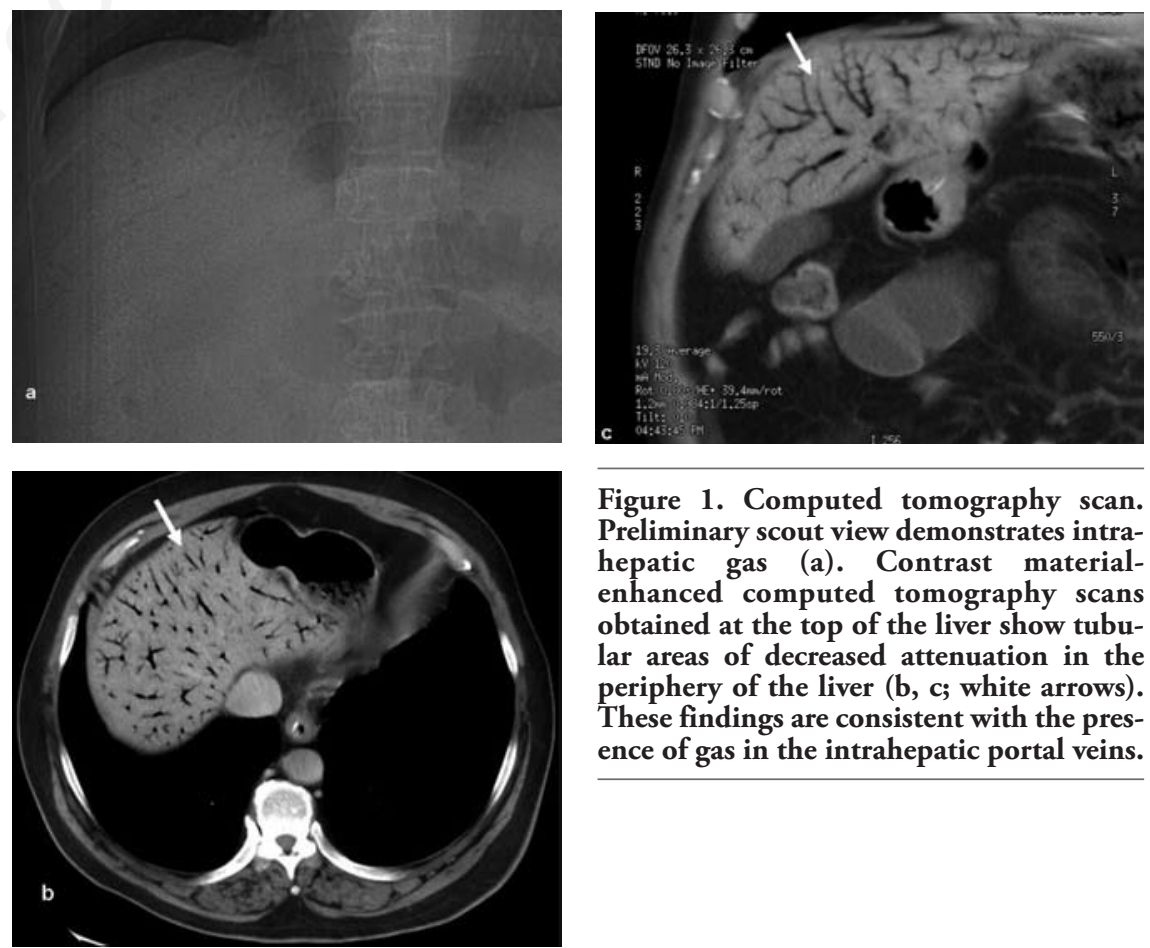

Figure 1. Computed tomography scan. Preliminary scout view demonstrates intrahepatic gas (a). Contrast materialenhanced computed tomography scans obtained at the top of the liver show tubular areas of decreased attenuation in the periphery of the liver (b, c; white arrows). These findings are consistent with the presence of gas in the intrahepatic portal veins. 


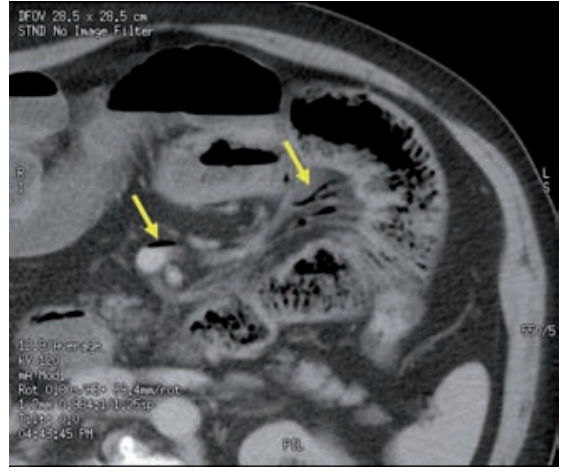

Figure 2. Contrast-enhanced computed tomography scan showing tubular or branched areas of decreased attenuation as expression of mesenteric vein gas (yellow arrows).

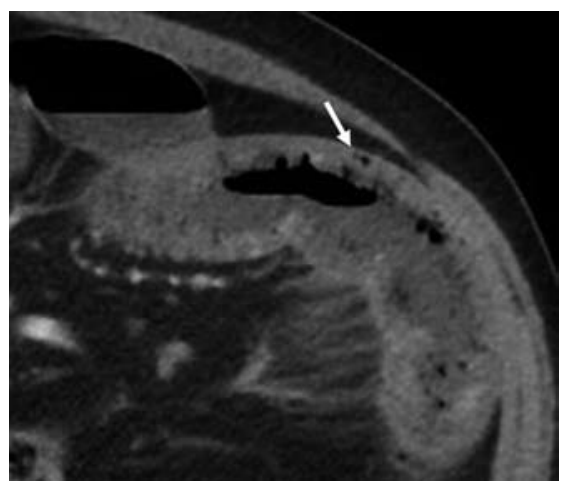

Figure 3. Bubble-like pneumatosis in small bowel. Enhanced computed tomography scan showing intramural bowel gas (white arrow).

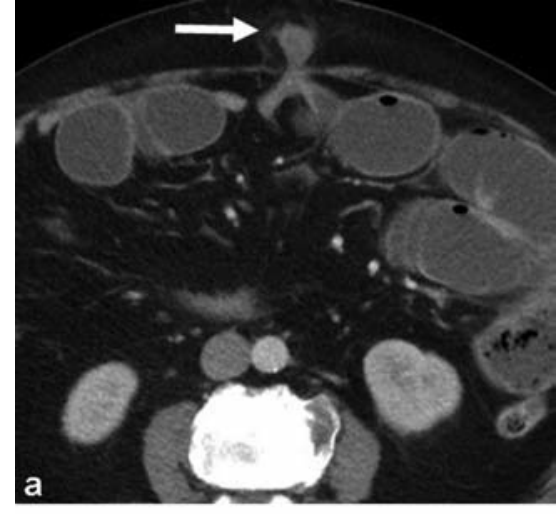

\section{References}

1. Plemmons RM, Dooley DP, Longfield RN. Septic thrombophlebitis of the portal vein: (pylephlebitis): diagnosis and management in the modern era. Clin Infect Dis 1995;21:1114-20.

2. Feczko PJ, Mezwa DG, Farab MC, White BD. Clinical significance of pneumatosis of the bowel wall. Radiographics 1992;12: 1069-78.

3. Wiesner W, Khurana B, Ji H, Ros PR. CT of acute bowel ischemia. Radiology 2003;226: $635-50$

4. Sebastià $\mathrm{C}$, Quiroga $\mathrm{S}$, Espin $\mathrm{E}$, et al. Portomesentericvein gas: pathologic mechanisms, CT findings, and prognosis. Radiographics 2000;20:1213-24.

5. Bradbury MS, Kavanagh PV, Bechtold RE, et al. Mesenteric venous thrombosis: diagnosis and noninvasive imaging. Radiographics 2002;22:527-41.

6. Wiesner W, Mortelé KJ, Glickman JN, et al. Pneumatosisintestinalis and portomesenteric venous gas in intestinal ischemia: correlation of CT findings with severity of ischemia and clinical outcome. Am J Roentgenol 2001;177:1319-23.

7. Lai WH, Hwang TL, Chen HW. Portomesenteric venous gas in acute bowel ischemia: report of a case. Surg Today 2008;38:656-60.

Figure 4. a) Humbilical hernia loop of the small intestine; b) sagittal view. White arrow in a) and b) indicates dilatation of the upstream part of small bowel.
8. García Figueiras R, Liñares Paz M, Baleato González S, Villalba Martín C. Case 158: pylephlebitis. Radiology 2010;255:1003-7. 